\title{
MEASUREMENT OF TWO-PHOTON ABSORPTION BY GOLD NANOPARTICLES OF DIFFERENT SIZES PHOTODEPOSITED ONTO THE CORE OF AN OPTICAL FIBRE
}

\author{
J.M. Cuvas-Limón a , J.G. Ortega-Mendoza ${ }^{\text {b }}$, J.P. Padilla-Martínez ${ }^{\mathrm{c}}$, and P. Zaca-Morán ${ }^{\mathrm{c}}$ \\ ${ }^{a}$ Facultad de Ciencias de la Electrónica, Benemérita Universidad Autónoma de Puebla, CP 72050, Puebla, México \\ ${ }^{\mathrm{b}}$ División de Posgrado, Universidad Politécnica de Tulancingo, CP 43629, Hidalgo, México \\ ' Instituto de Ciencias, Benemérita Universidad Autónoma de Puebla, CP 72050, Puebla, México \\ Email: zmoran_placido@icloud.com
}

Received 8 May 2020; revised 28 November 2020; accepted 1 December 2020

\begin{abstract}
In this work, the study of two-photon absorption by gold nanoparticles of different diameters photodeposited onto the core of a single-mode optical fibre is presented. The photodeposition of nanoparticles with diameters of 10 , 20,50 and $100 \mathrm{~nm}$ was achieved using a continuous wave laser at a wavelength of $1550 \mathrm{~nm}$ and a power of $50 \mathrm{~mW}$. Nonlinear optical characterization was carried out by using the P-scan technique of a high gain erbium doped fibre amplifier with pulses of $20 \mathrm{~ns}$ at a frequency of $10 \mathrm{kHz}$, that provides a maximum intensity of approximately $60 \mathrm{MW} / \mathrm{cm}^{2}$. The results show that for gold nanoparticles greater than $20 \mathrm{~nm}$ photodeposited onto the fibre, in both cases, the nonlinear coefficient as well as the third-order susceptibility increase as the diameter of the nanoparticles increases, describing a typical behaviour of the two-photon absorption. The obtained results can be used for the design of filters and optical limiters in the communications area.
\end{abstract}

Keywords: nonlinearity, optical fibres, gold nanoparticles, photodeposition

PACS: $42.65 . Y j, 42.81 .-\mathrm{i}, 81.07 .-\mathrm{b}$

\section{Introduction}

The study of nanostructured materials has attracted attention in several areas of science because they have different physicochemical properties in comparison with the bulk materials. This kind of materials is widely investigated for applications in optical storage, optical computing, harmonic generation, power limiting, rectifying devices and optical switching, therefore a high nonlinear response is a prerequisite [1-3]. Studies of the optical properties of nanomaterials, through interaction with high radiation intensities, have allowed one to know the dependence with its refractive index, giving rise to the phenomenon known as two-photon absorption (TPA) or saturable absorption (SA) [4, 5].
Currently TPA has been studied in new materials such as polymers [6], semiconductor materials [⿰], crystals [8], nanohybrid materials [9] and thin films [10], among others, which have been used in applications as optical limiters, light sensors, tunable emitters, etc. [11-13]. Reported studies on nonlinear effects have shown that gold nanoparticles (AuNPs) can behave as a TPA material, this response depends on their morphology $14-$ 16, the medium in which they are embedded [17], the volume fraction of AuNPs in solutions [10] and their proportion in a solution [18].

In 2015, Lysenko and co-workers presented the study of the dependence of third-order susceptibility on the thickness of wafer containing layers of deposited gold [19]. Their studies reported that 
the third-order susceptibility obtained depends on the thickness of the material and the susceptibility improves when the material is thinner. On the other hand, Maurya and co-workers published the study of TPA dependence of silver nanoparticles (AgNPs) and their concentration in a solution. The results show that the TPA predominates over the SA when the size of AgNPs is smaller, in addition to the SA being dominant over the TPA when the concentration is higher [20].

The present work gives a comparative study of the TPA at $1550 \mathrm{~nm}$ of AuNPs with different diameters photodeposited onto the core of a single-mode optical fibre. The nanoparticles were placed under the influence of an infrared radiation source and their nonlinear effects were studied using a high gain amplifier.

\section{Experiment}

Gold nanospheres were photodeposited onto the core of an optical fibre using the technique previously reported by a continuous wave laser source at $1550 \mathrm{~nm}$ and $50 \mathrm{~mW}$ as is shown in Fig. 1 (a) [21, 22]. AuNPs with diameters of 10, 20, 50 and $100 \mathrm{~nm}$ were acquired from Sigma-Aldrich company (741957, 741965, 742007 and 742031) and stabilized in a citrate buffer. The preparation of the optical fibre consisted of removing the plastic coating from the end of the fibre, cleaning it and making a cut on the fibre in the region where the nanospheres would be photodeposited. The fibre optic end was immersed into the solution with AuNPs, monitoring the photodeposition process until the transmission decreased to $3 \mathrm{~dB}$.
Figure 1(b) shows the experimental setup to carry out the nonlinear characterization of the fibre with AuNPs by P-scan technique. This setup is a high gain erbium doped fibre amplifier with two amplification stages that has been reported in [23]. In this arrangement, a pulse is emitted by a distributed feedback laser (DFB) that is controlled in current at $7.7 \mathrm{~mA}$ and stabilized in temperature at $5.2^{\circ} \mathrm{C}$ to emit at $1550 \mathrm{~nm}$. The square pulse of $20 \mathrm{~ns}$ is programmed at a frequency of $10 \mathrm{kHz}$. Under these conditions, it is possible to obtain an average power of $\sim 7 \mathrm{~mW}$ that corresponds to an irradiance of $\sim 60 \mathrm{MW} / \mathrm{cm}^{2}$, considering a single-mode optical fibre of $8 \mu \mathrm{m}$ core diameter. The preparation for each fibre with AuNPs consisted of splicing the opposite end of each sample in the end of the high gain amplifier; to carry out this process, it was necessary to remove the plastic coating from the opposite end of each fibre, clean it and make a cut in the splice area to then perform the fusion using a fusion splicer. Finally, the transmission behaviours for each sample were obtained by varying the input power.

\section{Theoretical calculations}

The theoretical study was carried out using the Beer-Lambert law [21, 22], that relates the attenuation of the intensity of radiation with the properties of the material by means of the transmission. In this work, the relationship between transmittance and absorbance is approximated by the expression

$$
T=\exp \left(-\left[\alpha_{0}+\beta \mathrm{I}\right] L\right),
$$

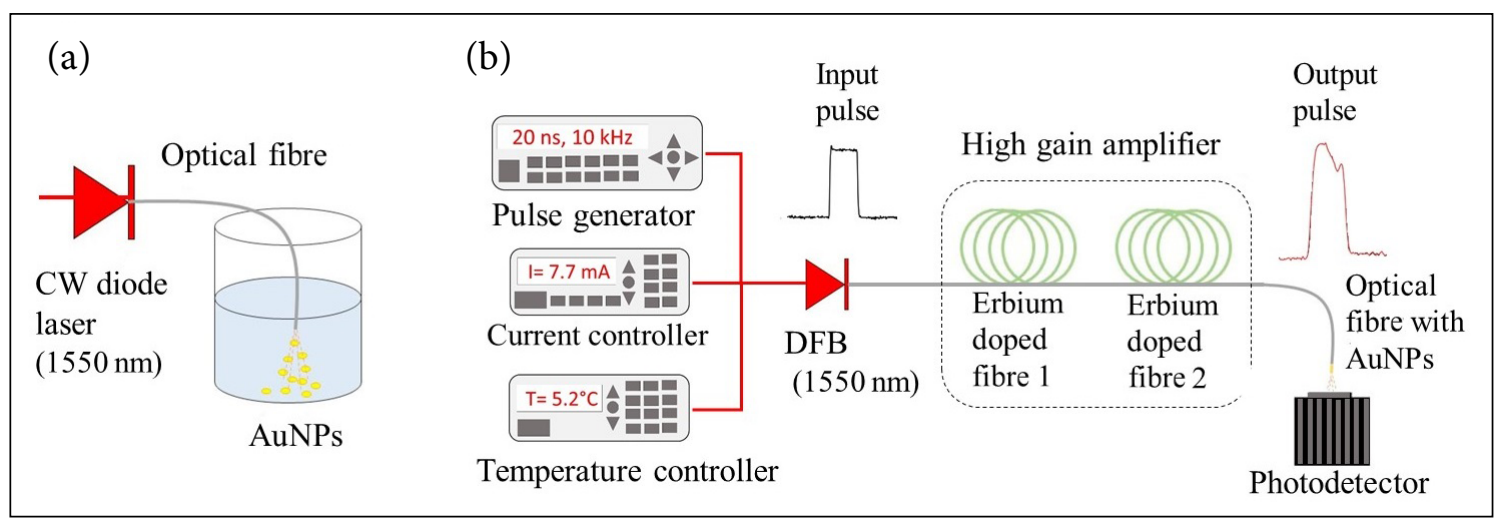

Fig. 1. Experimental setup of (a) the photodeposition technique of AuNPs onto the core of an optical fibre and (b) a high gain erbium doped fibre amplifier to carry out the nonlinear characterization. 
where $L$ is the thickness of the sample, $I$ is the intensity, $\alpha_{0}$ and $\beta$ are the linear and nonlinear absorption coefficients, respectively. Considering a saturation model, a hyperbolic approach is used to estimate the value of the absorption coefficient $\beta$,

$$
\beta(I)=\frac{\beta}{1+I / I_{\mathrm{sat}}},
$$

where $I_{\text {sat }}$ is the saturation intensity that is defined as the intensity when the transmission has reached $50 \%$ of the modulation. Combining the two equations we obtain the transmittance expression

$$
T=\exp \left[-\left(\alpha_{0}+\frac{\beta}{1+I / I_{\text {sat }}} I\right) L\right] .
$$

We can use Eq. (3) to fit the experimental data of P-scan trace, and $\beta$ can be treated as an adjustable parameter (Table 1). From the value of $\beta$, we can calculate the imaginary part of the third-order susceptibility by the following expression in the international system (SI) units,

$$
\operatorname{Im}\left(\chi^{(3)}\right)=\frac{\lambda \varepsilon_{0} n_{0}^{2} c \beta}{4 \pi},
$$

where $\lambda$ is the wavelength, $\varepsilon_{0}$ is the permittivity in free space, $c$ is the speed of light in vacuum, and $n_{0}$ is the refractive index of AuNPs [21]. We can convert the units of $\operatorname{Im}\left(\chi^{(3)}\right)$ in (esu) using the conversion formula $1(\mathrm{esu})=1.398 \times 10^{-8} \mathrm{~m}^{2} / \mathrm{V}^{2}$.

The study of the extinction coefficients for different diameters of the AuNPs was carried out theoretically by means of Mie analysis and experimentally by means of UV-Vis spectroscopy, using a Thermo Scientific Evolution 600 spectrometer with a resolution of $1 \mathrm{~nm}$ and in the total transmittance mode. In order to analyse the samples, the measurement region was from 400 to $800 \mathrm{~nm}$ :

$$
\begin{aligned}
& C_{\mathrm{ext}}=\frac{2 \pi r^{2}}{x^{2}} \sum_{l=1}^{\infty}(2 l+1) \operatorname{Re}\left(a_{l}+b_{l}\right), \\
& C_{\mathrm{sca}}=\frac{2 \pi r^{2}}{x^{2}} \sum_{l=1}^{\infty}(2 l+1)\left(\left|a_{l}\right|^{2}+\left|b_{l}\right|^{2}\right), \\
& C_{\mathrm{abs}}=C_{\mathrm{ext}}-C_{\mathrm{sca}} .
\end{aligned}
$$

Here $l$ denotes the multipolar order of the plasmons of excited particles, $x=2 \pi r / \lambda$ represents a size parameter proportional to the ratio of the radius of the sphere $r$ to the wavelength $\lambda$ for a wavelength of $1550 \mathrm{~nm}$ and the spheres with radii of 10, 20, 50 and $100 \mathrm{~nm}$. The coefficients of Mie are

$$
\begin{aligned}
& a_{l}=\frac{m \psi_{l}(m x) \psi_{l}^{\prime}(x)-\psi_{l}(x) \psi_{l}^{\prime}(m x)}{m \psi_{l}(m x) \xi_{l}^{\prime}(x)-\xi_{l}(x) \psi_{l}^{\prime}(m x)}, \\
& b_{l}=\frac{\psi_{l}(m x) \psi_{l}^{\prime}(x)-m \psi_{l}(x) \psi_{l}^{\prime}(m x)}{\psi_{l}(m x) \xi_{l}^{\prime}(x)-m \xi_{l}(x) \psi_{l}^{\prime}(m x)},
\end{aligned}
$$

expressed by the functions of Ricatti-Bessel $\psi_{l}$ and $\xi_{p}$, they contain the dependence of specific complex dielectric functions of the material through $\mathrm{m}^{2}=\varepsilon_{\text {metal }} / \varepsilon_{\text {medium }}$.

\section{Results and discussion}

The theoretical and experimental results for nanoparticles obtained by means of the UV-Vis and Mie analysis are shown in Fig. 2. Figures $2(a-d)$ show that the extinction coefficients are affected by their diameters. The theoretical results by Mie analysis show a shift of the wavelength towards the UV region when the diameter of the nanoparticle decreases. These results agree with the experimental data that show an absorption peak at longer wavelengths for nanoparticles with large diameters. This absorption peak also shows a shift towards the UV region for smaller nanoparticles. This behaviour is a well-known phenomenon that is attributed to the absorption by AuNPs, extinction coefficient and consequently to the effect of surface resonance plasmon (SPR) [21]. Studies have shown that the changes in the value of the SPR peak are due to the morphology, geometric dimensions and dielectric constants of a material [15, 24]. Additionally, it has been reported that in metals the SPR peak is sensitive to the size and amount of fraction of the particles that cause a change in the wavelength [25].

The nonlinear transmission as a function of irradiance is shown in Fig. 3. In this plot, the dotted lines indicate the transmittance obtained experimentally, and the solid lines indicate the transmittance obtained using the Beer-Lambert law. According to the results shown, it can be seen that when an optical fibre with AuNPs is irradiated by 


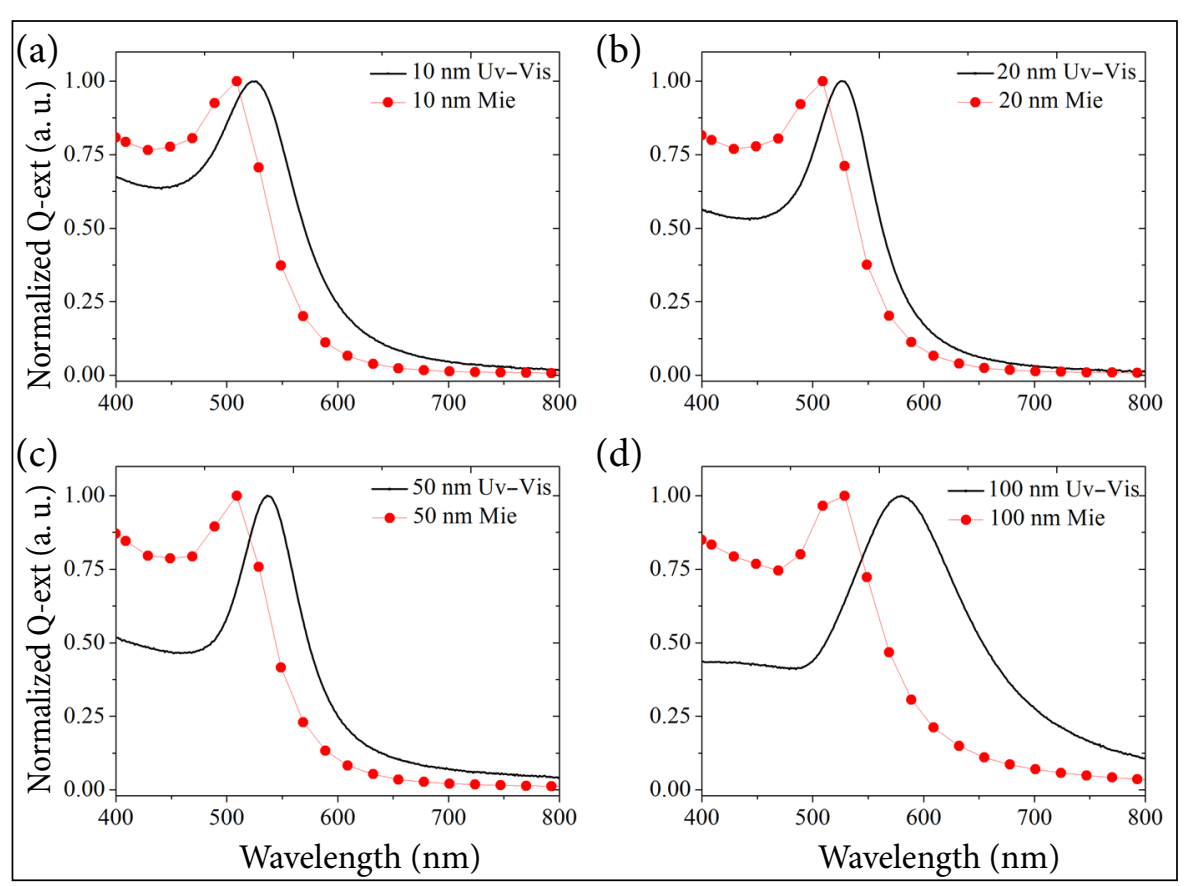

Fig. 2. Extinction coefficients as a function of the wavelength of AuNPs with diameters of (a) $10 \mathrm{~nm}$, (b) $20 \mathrm{~nm}$, (c) $50 \mathrm{~nm}$ and (d) $100 \mathrm{~nm}$.

the $20 \mathrm{~ns}$ pulses of $1550 \mathrm{~nm}$ laser source, the transmittance decreases linearly in the region of low irradiance; on the other hand, the results show a decrease in transmission as intensity increases, showing that the AuNPs of $10 \mathrm{~nm}$ have the lowest level of transmission and the AuNPs $100 \mathrm{~nm}$ have the highest level.
Figure 4 depicts the nonlinear transmission behaviours of each sample as a function of irradiance using the Beer-Lambert law. The results show that the decrease in the transmission at low intensities for each nanoparticle size has different saturation intensities. The nanoparticles of 10, 20, 50 and $100 \mathrm{~nm}$ show saturation intensities of 8.2,

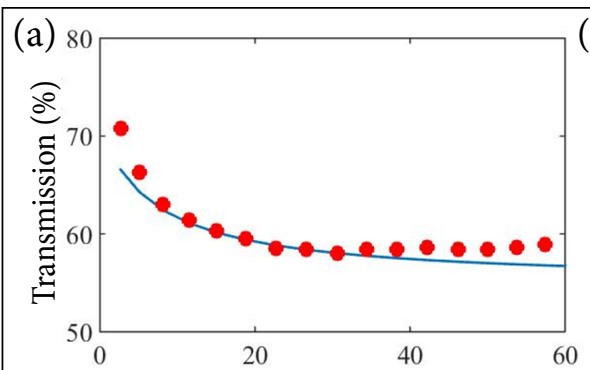

(c)
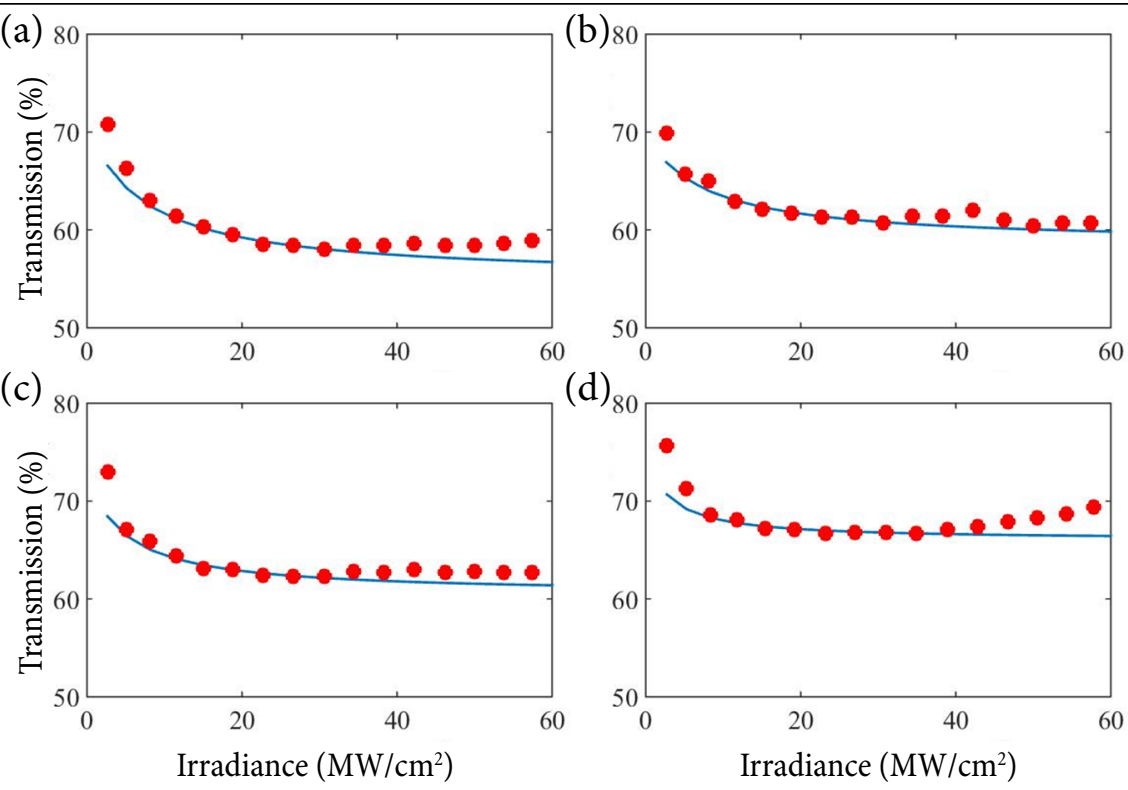

(d) 80

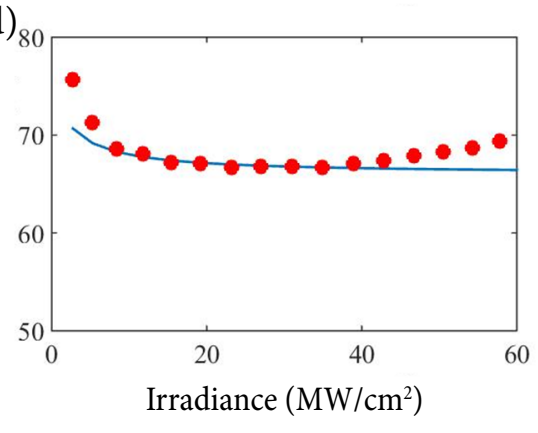

Fig. 3. Transmission as a function of the irradiance of the AuNPs of (a) $10 \mathrm{~nm}$, (b) $20 \mathrm{~nm}$, (c) $50 \mathrm{~nm}$ and (d) $100 \mathrm{~nm}$. 


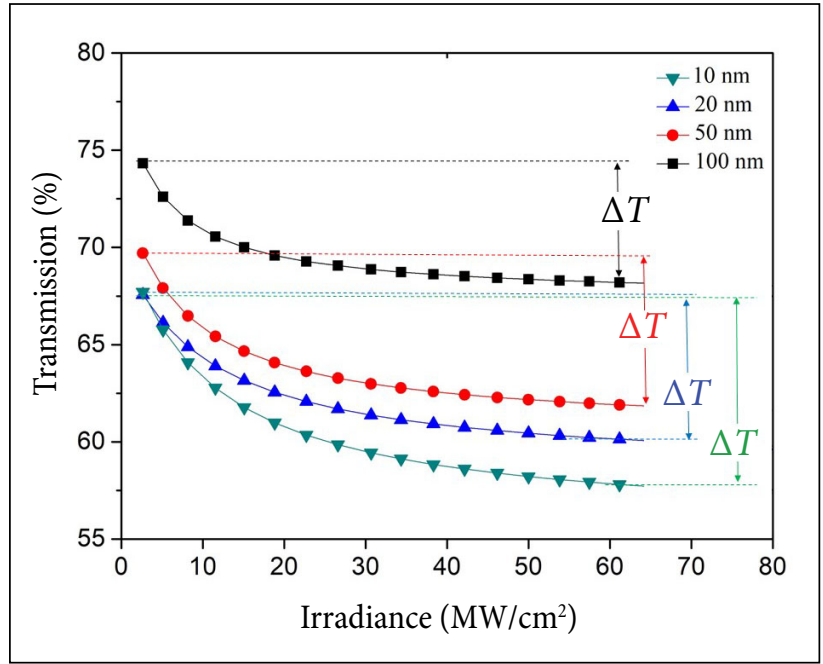

Fig. 4. Transmission adjustment based on the irradiance of AuNPs.

8.1, 5.1 and $2.7 \mathrm{~W} / \mathrm{cm}^{2}$, respectively, implying that small AuNPs have the ability to gradually absorb more intense amounts of radiation before saturating. This optical limiting mechanism is attributed to the combination of laser induced AuNPs dispersion and the nonlinear absorption. In addition, it can be observed that the saturation intensity and the modulation depth $(\Delta T)$ increase for smaller AuNPs diameters, these results are due to the $I_{\text {sat }}$ greater magnitude. Consequently the linear absorption coefficient $\alpha_{0}$ increases, respectively, as shown in Table 1. This dependence of the depth of modulation on the diameter of the sphere, that is inversely proportional, indicates that it can be used for the design of lasers, optical limiters and light sensors, among others. All the results show a typical behaviour of the TPA curve [21].

In addition, the results show that for AuNPs greater than $20 \mathrm{~nm}$ the nonlinear coefficient $\beta$ and third-order susceptibility increase as the diameter of the nanoparticles increases, that indicates that the TPA improves since its capacity of optical limi- tation increases [2]. Optical limiting occurs when the optical transmission of a material decreases with increasing laser fluence, a property that is desirable for protection of sensors and human eyes from intense laser radiation.

On the other hand, it is important to mention that the advantage of using the technique of photodeposition of nanostructured materials on the nucleus of optical fibres is the minimal use of nanostructured materials, in contrast to the devices made on films, crystals or solutions. This technique allows one, as it has been demonstrated, to have a better control of the nonlinear parameters of the materials placed on the fibres, that makes it ideal for the development of photonic devices. Clearly, the AuNPs endowed the fibres with a limiting power due to the optical limiting mechanisms for AuNPs that are quite different from those of carbon nanotubes.

\section{Conclusions}

The AuNPs photodeposited onto the core of a single-mode optical fibre exhibit nonlinear properties depending on the dimension of their diameters. Maintaining a constant transmission in each fibre sample with AuNPs, the nonlinear optical characterization was performed for diameters between 10 and $100 \mathrm{~nm}$. The results obtained in our experiments showed a typical behaviour of two-photon absorption, achieving saturation when the intensity increased. Additionally, it was observed that the modulation depth turned out to be greater when the diameter of the gold nanoparticle was smaller. To our knowledge, the study of the nonlinear dependence of this type of nanomaterials photodeposited on an optical fibre has not been reported, which can be used for the implementation of photonic devices based on nanostructured materials and optical fibres.

Table 1. Absorption coefficients and third-order susceptibility of the different diameters of AuNPs.

\begin{tabular}{cccccc}
\hline Diameter $(\mathrm{nm})$ & $\alpha_{0}(1 / \mathrm{m})$ & $\Delta T(\%)$ & $\beta(\mathrm{m} / \mathrm{W}) \times 10^{-6}$ & $\chi^{(3)}(\mathrm{esu}) \times 10^{-7}$ \\
\hline 10 & 3.055 & 11.48 & 3.084 & 1.9810 \\
\hline 20 & 3.588 & 10.16 & 2.152 & 1.3824 \\
\hline 50 & 3.153 & 10.14 & 3.659 & 2.3500 \\
\hline 100 & 2.794 & 7.97 & 4.956 & 3.1832 \\
\hline
\end{tabular}




\section{Acknowledgements}

This research was supported by Fondo Institucional del Conacyt (FOINS) under Grant No. 2319.

\section{References}

[1] Y. Golian and D. Dorranian, Effect of thickness on the optical nonlinearity of gold colloidal nanoparticles prepared by laser ablation, Opt. Quant. Electron. 46, 809-819 (2014), https://doi. org/10.1007/s11082-013-9792-2

[2] C. Li, Y. Qi, X. Hao, X. Peng, and D. Li, Gold nanorods-silicone hybrid material films and their optical limiting property, Appl. Phys. A 121, 11-15 (2015), https://doi.org/10.1007/s00339-015-9401-Z

[3] N. Vijayan, G. Bhagavannarayana, S.K. Halder, S. Verma, J. Philip, R. Philip, and B. Rathi, X-ray topography, photopyroelectric and two-photon absorption studies on solution grown benzimidazole single crystal, App. Phys. A 110, 55-58 (2013), https://doi.org/10.1007/s00339-012-7448-7

[4] O.M. Bankole and T. Nyokong, Mercatopyridinesubstituted indium, zinc, and metal-free phthalocyanines: nonlinear optical studies in solution and on polymer matrices, J. Coord. Chem. 68, 3727-3740 (2015), https://doi.org/10.1080/00958 972.2015 .1077237

[5] K.V. Anand, G. Vinitha, M.K. Chinnu, R. Mohan, and R. Jayavel, Enhanced third-order nonlinear optical properties of high purity $\mathrm{ZnS}$ nanoparticles, J. Nonlinear Opt. Phys. Mater. 24(02), 1-11 (2015), https://doi.org/10.1142 \$0218863515500162

[6] M.S. Sunitha, K.A. Vishnumurthy, and A.V. Adhikari, Synthesis and two-photon absorption property of new $\pi$-conjugated donor-acceptor polymers carrying different heteroaromatics, J. Chem. Sci. 125, 29-40 (2013), https://doi.org/10.1007 s12039-013-0366-1

[7] P.P. Kiran, S.V. Rao, M. Ferrari, B.M. Krishna, H. Sekhar, S. Alee, and D.N. Rao, Enhanced optical limiting performance through nonlinear scattering in nanoparticles of CdS, co-doped Ag$\mathrm{Cu}$, and BSO, Nonlinear Opt. Quantum Opt. 40, 223-234 (2010).
[8] V.I. Lukanin, D.S. Chunaev, and A.Y. Karasik, Two-photon absorption of high-power picosecond pulses in $\mathrm{PbWO}_{4}, \mathrm{ZnWO}_{4}, \mathrm{PbMoO}_{4}$, and $\mathrm{CaMoO}_{4}$ crystals, J. Exp. Theor. Phys. 113(3), 412-421 (2011), https://doi.org/10.1134 S1063776111070077

[9] Q. Zhang, X. Tian, H. Zhou, J. Wu, and Y. Tian, Lighting the way to see inside two-photon absorption materials: structure-property relationship and biological imaging, Materials 10(3), 1-37 (2017), https://doi.org/10.3390/ma10030223

[10]R.A. Ganeev, A.I. Ryasnyanskii, M.K. Kodirov, S.R. Kamalov, and T. Usmanov, Optical limiting in fullerenes, colloidal metal solutions, and semiconductors in the field of pico- and nanosecond pulses of an Nd:YAG laser, Opt. Spectrosc. 93(5), 789-796 (2002), https://doi. org/10.1134/1.1524003

[11]S. Hirata, K. Totani, T. Yamashita, C. Aadachi, and $\mathrm{M}$. Vacha, Large reverse saturable absorption under weak continuous incoherent light, Nat. Mat. 13, 938-946 (2014), https://doi.org/10.1038/ nmat4081

[12] R.A. Silva and M.O. Orlandi, Influence of synthesis route on the radiation sensing properties of $\mathrm{ZnO}$ nanostructures, J. Nanomater. 2016, 1-9 (2016), https://doi.org/10.1155/2016/4054058

[13]L. Irimpan, V.P.N. Nampoori, and P. Radhakrishnan, Spectral and nonlinear optical characteristics of $\mathrm{ZnO}$ nanocomposites, Sci. Adv. Mater. 2(2), 117-137 (2010), https://doi.org/10.1166

[14]S. Kumar, M. Anija, and A.K. Sood, Tuning ultrafast photoresponse of gold nanorods, Plasmonics 8, 1477-1483 (2013), https://doi.org/10.1007 s11468-013-9561-7

[15]T. Cesca, P. Calvelli, G. Battaglin, P. Mazzoldi, and G. Mattei, Nonlinear optical response of gold-silver nanoplanets, Radiat. Eff. Defect. S. 167(7), 520-526 (2012), https://doi.org/10.1080/1042015 0.2012 .680458

[16]H.I. Elim, J. Yang, and J.Y. Lee, Observation of saturable and reverse-saturable absorption at longitudinal surface plasmon resonance in gold nanorods, Appl. Phys. Lett. 88(8), 083107 (2006), https://doi.org/10.1063/1.2177366 
[17]Y. Yang and M. Nogami, Controlled surfaceplasmon coupling in $\mathrm{SiO}_{2}$-coated gold nanochains for tunable nonlinear optical properties, Appl. Phys. Lett. 88, 081110 (2006), https://doi. org/10.1063/1.2172022

[18]J. Zhu, S.W. Bai, J.W. Zhao, and J.J. Li, Tunable optical limiting of gold nanorod thin films, Appl. Phys. A 97, 431-436 (2009), https://doi. org/10.1007/s00339-009-5232-0

[19] O. Lysenko, M. Bache, and A. Lavrinenko, Thirdorder susceptibility of gold for ultrathin layers, Opt. Lett. 41(2), 317-320 (2016), https://doi. org/10.1364/OL.41.000317

[20]S.K. Maurya, A. Rout, R.A. Ganeev, and C. Guo, Effect of size on the saturable absorption and reverse saturable absorption in silver nanoparticle and ultrafast dynamics at $400 \mathrm{~nm}$, J. Nanomater. 2019, 1-12 (2019), https://doi. org/10.1155/2019/9686913

[21]P. Zaca-Morán, R. Ramos-Garcia, J.G. OrtegaMendoza, F. Chávez, G.F. Pérez-Sánchez, and C. Felipe, Saturable and two-photon absorption in zinc nanoparticles photodeposited onto the core of an optical fiber, Opt. Express 23(14), 18721-18729 (2015), https://doi.org/10.1364 OE.23.018721

[22]L.C. Gómez-Pavón, G.J. Lozano-Perera, A. LuisRamos, J.M. Muñoz-Pacheco, J.P. Padilla-Mar- tínez, and P. Zaca-Morán, Influence on the saturable absorption of the induced losses by photodeposition of zinc nanoparticles in an optical fiber, Opt. Express 26(2), 1556-1563 (2018), https://doi.org/10.1364/OE.26.001556

[23]P. Zaca-Morán, E. Kuzin, J. Torres-Turiján, J.G. Ortega-Mendoza, F. Chávez, G.F. PérezSánchez, and L.C. Gómez-Pavón, High gain pulsed erbium-doped fiber amplifier for the nonlinear characterization of SWCNTs photodeposited on optical fibers, Opt. Laser Technol. 52, 15-20 (2013), https://doi.org/10.1016/j.optlastec.2013.04.004

[24]A. Alesenkov, J. Pilipavičius, A. Beganskienè, R. Sirutkaitis, and V. Sirutkaitis, Nonlinear properties of silver nanoparticles explored by a femtosecond Z-scan technique, Lith. J. Phys. 55(2), 100-109 (2015), https://doi.org/10.3952/physics. v55i2.3100

[25]S. Mohapatra, Y.K. Mishra, A.M. Warrier, R. Philip, S. Sahoo, A.K. Arora, and D.K. Avasthi, Plasmonic, low frequency Raman, and nonlinear optical-limiting studies in copper-silica nanocomposites, Plasmonics 7, 25-31 (2012), https:// doi.org/10.1007/s11468-011-9271-y

\title{
FOTODEPOZICIJOS BŪDU ANT OPTINIO ŠVIESOLAIDŽIO ŠERDIES NUSODINTŲ AUKSO DALELIŲ DVIFOTONĖS ABSORBCIJOS MATAVIMAI
}

\author{
J.M. Cuvas-Limón a , J.G. Ortega-Mendoza ${ }^{\text {b }}$,.P. Padilla-Martínez ${ }^{\text {c }}$, P. Zaca-Morán c \\ a Autonominio Pueblos universiteto Elektronikos fakultetas, Puebla, Meksika \\ ${ }^{\mathrm{b}}$ Tulancingo politechnikos universitetas, Hidalgas, Meksika \\ ${ }^{c}$ Autonominio Pueblos universiteto Mokslo institutas, Puebla, Meksika
}

